\title{
Explicit solutions for the design of initially deformed plates subject to compression
}

\author{
D. G. WILLIAMS \& A. C. WALKER
}

\begin{abstract}
Dr P. A. Frieze, Department of Civil Engineering. Imperial College of Science and Technology
\end{abstract}

Presumably when discussing mesh sizes in relation to the first-order finite difference approximations of the derivatives in the von Karman large deflexion differential equations $(\$ 9)$ the Authors were referring to the dynamic relaxation part of their combined approach. I have found ${ }^{24}$ that for the application of dynamic relaxation to similar plate problems an $8 \times 8$ interlacing mesh gives satisfactory convergence even in the elasto-plastic range. As the Authors required a $12 \times 12$ mesh to achieve a satisfactory degree of accuracy, have they used a non-interlacing mesh? If so, it appears that such an arrangement suffers considerably in both computer storage and time compared with the interlacing mesh I used.

34. I have studied the elasto-plastic behaviour of plates in compression ${ }^{25,28}$ and from the data the following results were obtained in connexion with the first example presented in the Paper

$$
\begin{aligned}
\text { collapse stress } & =307 \mathrm{~N} / \mathrm{mm}^{2} \\
\text { factor against collapse } & =307 / 200=1.54
\end{aligned}
$$

where $200 \mathrm{~N} / \mathrm{mm}^{2}$ is the average applied stress. These values apply only in the case where the box column can be considered as stocky. For more slender columns overall buckling is possible before the maximum plate strength is developed because of the loss of plate in-plane stiffness that follows yielding. In the case where interactive buckling of the plate and column can occur, both plate strength and in-plane secant stiffness (effective width) are required and have been obtained as $^{26}$

$$
\begin{aligned}
\text { maximum allowable average stress } & =304 \cdot 5 \mathrm{~N} / \mathrm{mm}^{2} \\
\text { factor against collapse } & =304 \cdot 5 / 200=1 \cdot 52 \\
\text { in-plane secant stiffness } & =0.92
\end{aligned}
$$

35. Comparing the factors against collapse with the membrane yield factor given in the Paper indicates that my elasto-plastic analysis possibly gives estimates of plate strength which are conservative in relation to those of the Authors.

36. In following the examples in the Paper it was noticeable that some experience appeared necessary for establishing the initial value of $w / t$. The subsequent workings showed that convergence to the correct value of the ratio of deflexion to plate thickness was very sensitive to this parameter which could prove a hindrance in applying the procedure in practice.

37. It appears from the first example that the extent of the calculations is far greater than is necessary using my method for establishing the strength and stiffness of uniaxially

Paper published: Proc. Instn Civ. Engrs, Part 2, 1975, 59, Dec., 763-787. 
compressed plates. ${ }^{25.26}$ However, it is appreciated that the generality of the Authors' results probably necessitates such an approach.

Mr D. Parry, British Ship Research Association

There is no mention in the Paper of the manner in which the plate deflects, except that it is defined by a power series. It can be deduced from example 2 that the deflected shape takes the form of one half sine wave in each principal direction irrespective of the aspect ratio of the plate. One might ask, "What is inhibiting the plate of aspect ratio 3 having a deflected shape in the form of three half sine waves in the longitudinal direction?'

39. The analytical approach used, adopting a power series for the intrinsic problem parameters, seems to be analogous to the assumption of the classical sine wave out-ofplane deformation if one considers the sine function expanded in a power series. The use of the first two terms of the expanded sine series would probably give accurate agreement with precise numerical solutions for the post-buckling of the plate, with the deflexion being symmetrical with respect to the loading. Hence it can be concluded that in this analytical approach the finite difference technique is used to adjust the first two coefficients of a sine series to incorporate the remainder of the series. Therefore, with such a similarity of approach to the classical solution, the adoption of only one half sine wave lateral deflexion for aspect ratios greater than one seems to be questionable.

\section{Dr Williams and Dr Walker}

Dr Frieze is correct in presuming that the references to mesh size relate to the numerical solution part of our approach. The question of convergence is discussed in more detail in reference 21 . Table 7 , taken from reference 21 , gives some idea of the convergence achieved. A single mesh grid was used for all variables.

41. Without more detailed knowledge of what Dr Frieze considers a satisfactory degree of accuracy it is difficult to make further comment. Certainly, from a design viewpoint, it could be argued that the convergence of maximum membrane stress given in Table 7 is satisfactory for an $8 \times 8$ mesh, but corner twisting moment is not. On the relative merits of interlacing as opposed to non-interlacing mesh we are aware that in general interlacing mesh converges more rapidly but it does require interpolation for variables defined on one or other of the mesh systems on the boundary. In view of the sensitivity of corner values we doubt that there is significant advantage.

42. We find the degree of agreement between Dr Frieze's factor against collapse of 1.54 (1.52 allowing interaction) using elastic-plastic theory and our approximate mem-

Table 7. Dynamic relaxation solutions for square simply supported plate subject to uniaxial compression, unloaded sides stress free $\left(w_{0} / t=0.25\right)$

\begin{tabular}{c|c|c|c|c}
\hline $\begin{array}{c}\text { Mesh } \\
\text { intervals }\end{array}$ & $\begin{array}{c}\text { Maximum } \\
\text { deflexion } \\
w / t\end{array}$ & $\begin{array}{c}\text { Corner } \\
\text { membrane stress } \\
\sigma_{x 1} / \sigma_{x u \mathrm{v}}\end{array}$ & $\begin{array}{c}\text { Maximum } \\
\text { membrane stress } \\
\sigma_{x 2} / \sigma_{x \mathrm{av}}\end{array}$ & $\begin{array}{c}\text { Corner } \\
\text { twisting moment } \\
M_{x y}\left(b^{2} / E t^{4}\right)\end{array}$ \\
\hline $6 \times 6$ & 0.520 & $\mathbf{1 . 1 4}$ & 1.62 & 0.407 \\
$8 \times 8$ & 0.492 & $\mathbf{1 . 1 1}$ & 1.66 & 0.363 \\
$10 \times 10$ & 0.482 & 1.09 & 1.69 & 0.348 \\
$12 \times 12$ & 0.476 & 1.06 & 1.70 & 0.340 \\
$14 \times 14$ & 0.472 & 1.05 & 1.71 & 0.336 \\
$16 \times 16$ & 0.470 & 1.04 & 1.71 & 0.334 \\
$18 \times 18$ & 0.470 & 1.04 & 1.72 & 0.332 \\
\hline
\end{tabular}


brane yield factor of 1.59 most encouraging. Dr Frieze's analysis is undoubtedly more accurate in the elasto-plastic range but the correlation confirms that a membrane yield criterion gives a reasonable design estimate of collapse strength.

43. The design relevance of in-plane secant stiffness at collapse is not immediately apparent. We believe that the value of 0.95 quoted in the example for in-plane stiffness at the design load level is the required design stiffness criterion in this case, as local yielding has not begun.

44. We anticipate that our explicit expressions will generally be used as part of an automated design process in which case Dr Frieze's concern regarding the necessity of experience in establishing initial values of $w / t$ and sensitivity of convergence need not arise. In cases where they are used by hand the designer will rapidly develop a feel for the problem.

45. We are not conversant with Dr Frieze's method of calculating the strength and stiffness of uniaxially compressed plates so we cannot comment on the extent of the required calculations. The computation required to derive maximum membrane stress and stiffness using the explicit expressions is not, we believe, unduly onerous and the question does not arise when the process is automated. Various other required design parameters are computed as part of the same exercise which we presume are not catered for by Dr Frieze's approach. The complexity can be further reduced by using the one term solutions given in the Appendix for square plates. We assume that because Dr Frieze's analysis is based on a finite element elasto-plastic program it will be necessary to have a separate run on the computer for each particular geometry loading condition and imperfection level. In fact the reason for developing the approach in the Paper was to enable such computer based analysis to be presented in a simple and more readily usable form.

46. It would be instructive to extend the comparison between Dr Frieze's more exact collapse calculations and our approximate solutions so as to provide guidelines on the accuracy of this part of our suggested design procedure.

47. With reference to Mr Parry's remarks, the form of the initial imperfections is as noted in $\S 12$, the appropriate primary critical mode shape for each load case. This corresponded to a single half sine wave in all the cases tablulated and the solutions maintained this basic deflected form under load. Secondary modes develop as the load increases but in no case did the solution jump into a higher critical mode.

48. In practice, initial imperfections will not necessarily be in the form of the 'preferred ' primary mode but the single half wave forms can occur even for longer plates. Hence the primary mode should be the design criterion because it will generally give rise to higher secondary stresses than any other mode shape. There are exceptions to this, namely cases where the plate jumps to a higher mode at applied loads in excess of the lowest critical load. None of the tabulated solutions showed any tendency to do this but an example would be a long plate loaded in the uniaxial direction. In such cases it has been found that the numerical solution will jump into alternate higher modes maintaining an even or odd half waveform corresponding to the number of half waves in the primary node. This is unlikely to be a practical design consideration for plates subject to compressive loading.

49. With regard to the nature of the solution represented by substituting the numerical results to define the coefficients of a two term series, it is not a question of adjusting coefficients to incorporate higher terms but rather of deriving coefficients for solutions which cannot be solved analytically. The numerical solutions of course incorporate all modes within the limits of the accuracy of the mathematical model, and therefore there is an intrinsic inconsistency in substituting the results into a series which can define only the first mode. This error becomes more apparent as the load increases; hence the comparison made in Figs 2-4 to evaluate the significance of the error. It was concluded that the accuracy achieved by the two term solutions was satisfactory for design purposes although, as noted in $\S 15$, we are considering including more terms for certain variables. 
DISCUSSION

\section{References}

24. FRIEZE P. A. Ultimate load behaviour of steel box girders and their components. PhD thesis, University of London, 1975.

25. Frieze P. A. et al. Steel box girders. Parametric study on plates in compression. Engineering Structures Laboratories, Civil Engineering Department, Imperial College, London, CESLIC report BG39, 1975.

26. FriezE P. A. et al. Steel box girders. A contribution to the design of stiffened compression flanges. Engineering Structures Laboratories, Civil Engineering Department, Imperial College, London, CESLIC report BG42, 1976. 